\title{
In-Patient Treatment of Chronic Daily Headache Using Dihydroergotamine: a Long-term Follow-up Study
}

\author{
Tamara Pringsheim and David Howse
}

\begin{abstract}
Background: The treatment of chronic daily headache (CDH) due to medication overuse remains a common and difficult problem. For selected patients refractory to outpatient management we have used a treatment protocol using dihydroergotamine (DHE) as introduced by Raskin, during a brief (typically 48 hours) in-patient stay. While many studies have documented the short-term efficacy of the DHE protocol, there are limited data on its long-term effects. The purpose of this study was to evaluate the efficacy of the protocol on headache frequency and severity, analgesic use, absences from work, and quality of life, at three months post treatment and the present time. Methods: A retrospective chart review of all patients admitted for the DHE protocol from 1991 to 1996 revealed 174 cases. Of these, 132 patients were interviewed by phone. Results: The DHE protocol was shown to decrease headache frequency, severity, headache medication use, and absences from work both at three months and the time of interview. Conclusion: This study has the largest patient base and the longest follow-up period for the use of DHE for CDH. The results confirm that the DHE protocol is helpful in breaking the cycle of CDH, although the long-term outcomes of this study are more conservative than other studies have reported.
\end{abstract}

\begin{abstract}
RÉSUMÉ: Traitement hospitalier de la céphalée quotidienne chronique au moyen de la dihydroergotanine: une étude à long terme. Introduction: Le traitement de la céphalée quotidienne chronique (CQC) due à la surutilisation médicamenteuse demeure un problème fréquent et difficile. Pour des patients externes bien choisis, réfractaires au traitement en externe, nous avons utilisé le protocole de Raskin utilisant la dihydroergotamine (DHE) au cours d'un court séjour hospitalier (habituellement de 48 heures). Bien que plusieurs études ont montré l'efficacité à court terme du protocole DHE, il existe peu de données sur les résultats à long terme. Le but de cette étude était d'évaluer l'efficacité du protocole quant à la fréquence et la sévérité des céphalées, l'utilisation d'analgésiques, l'absentéisme au travail et la qualité de vie trois mois après le traitement et jusqu'au moment de l'étude. Méthodes: Une revue rétrospective des dossiers de tous les patients admis pour traitement au moyen du protocole DHE de 1991 à 96 a indiqué qu'il y avait eu 174 cas ainsi traités. Parmi ceux-ci, 132 patients ont eu une entrevue téléphonique. Résultats: Le protocole DHE a diminué la fréquence des céphalées, leur sévérité, la prise de médicaments et l'absentéisme au travail à 3 mois et au moment de l'entrevue. Conclusion: Cette étude inclus le plus grand nombre de patients et le suivi le plus long après l'utilisation de la DHE dans les cas de CQC. Ces résultats confirment que le protocole DHE aide à briser le cycle de la $\mathrm{CQC}$, même si les résultats à long terme rapportés dans cette étude sont plus conservateurs que ceux rapportés dans d'autres études.
\end{abstract}

Can. J. Neurol. Sci. 1998; 25: 146-150

Chronic daily headache $(\mathrm{CDH})$ is a difficult clinical problem, affecting approximately $0.5 \%$ of the population. ${ }^{1} \mathrm{CDH}$ affects 40 to $75 \%$ of patients ${ }^{2}$ seen at major headache clinics, and is a poorly understood entity both in pathophysiology and in management. CDH may be perpetuated by the use of symptomatic medications for headache relief if used daily or almost daily. Other types of $\mathrm{CDH}$ include transformed migraine, chronic tension type headache, and new daily persistent headache. ${ }^{2.3}$

Treatment of CDH is difficult and often ineffective. The current standard of practice involves: (1) discontinuation of offending headache medications, (2) administration of pharmacotherapeutic agents in an attempt to break the cycle of continuous headache, and (3) initiation of prophylactic medications. ${ }^{2.4}$ A study performed by Raskin in 1986 showed that repetitive intravenous dihydroergotamine can break the cycle of
$\mathrm{CDH}$ in an in-patient setting. ${ }^{5}$ Dihydroergotamine, a derivative of ergotamine tartrate, is a presynaptic $5 \mathrm{HT}_{1}$ agonist of the trigeminal vascular system, and is thought to inhibit the release of neuropeptides, reducing vasodilation and inflammation of cranial vessels. ${ }^{6.7}$

While the short-term efficacy of in-patient DHE therapy has been documented, ${ }^{5,8}$ there are comparatively few studies on the long-term outcome. At the Kingston General Hospital, we have used a modified form of Raskin's protocol to treat patients with

From the Department of Neurology, Queen's University, Kingston, Canada. RECIEVED AUGUST 11, 1997. ACCEPTED IN FINAL FORM NOVEMBER 13, 1997. Reprint requests to: David Howse, Richardson House, 102 Stuart Street, Kingston, Ontario, Canada K7L 2V6 
$\mathrm{CDH}$ in an in-patient setting since 1991. The purpose of this study is to evaluate the long-term effects of the DHE protocol on patients at our centre, by evaluating headache frequency and intensity, headache medication use, absences from work, and quality of life, before treatment, three months after treatment, and at the time of interview.

\section{METHODS}

All patients were referred by one of six staff neurologists on the basis of failure of various standard outpatient regimes. All these patients had $\mathrm{CDH}$, and most were taking considerable amounts of analgesic medications, migraine prophylactics, and acute migraine therapies. Although the evolution of $\mathrm{CDH}$ in each patient was not determined for this study, the majority of patients had $\mathrm{CDH}$ due to transformed migraine. A retrospective chart review of patients admitted to the Kingston General Hospital for the DHE protocol from 1991 to April 1996 revealed 174 patients. We attempted to contact all patients from July to October 1996. Of these, 132 patients were interviewed by telephone. The remaining 42 patients were unable to be contacted due to wrong phone numbers, or out-of-service numbers, and were therefore lost to follow-up.

Our standardized DHE protocol consists of (1) cessation of all analgesic and ergot medications (2) 10 to $20 \mathrm{mg}$ of metoclopramide IV thirty minutes prior to each DHE dose (3) 0.5 to 1.0 $\mathrm{mg}$ of DHE through saline infusion over twenty minutes. The times of DHE administration are standardized at $0730 \mathrm{~h}, 1200 \mathrm{~h}$, $1800 \mathrm{~h}, 2400 \mathrm{~h}$. Patients are monitored after each administration of DHE for chest pain, nausea, sensations in the extremities, and any other adverse reactions. Most patients were discharged on a preventive headache medication, given instructions to avoid using analgesics for a period of two weeks after treatment and told to restrict future analgesic use to only the most severe headaches.

The questionnaire consisted of 25 questions about headache frequency and intensity, headache medication use, and absences from work, which were asked concerning the period of time prior to the DHE protocol, three months after the DHE protocol, and at the time of interview. Additional questions assessed the patient's course in hospital - their response to treatment and any complications. Improvements or worsening of the patient's quality of life after the DHE protocol were also assessed, as well as changes in the amount of money spent on headache medications. Patients also answered questions regarding depression, visits to the emergency room because of headaches, and the impact of DHE treatment.

\section{RESULTS}

112 of the 132 patients interviewed were women. 128 patients had chronic daily headache or near-daily headache for at least six months, and in some patients for several years. All patients had failed to respond to outpatient therapy. Two patients were admitted through the emergency room for headaches of two weeks or longer. Another two patients had migraines less than once a week, but were considered of great enough severity and morbidity to allow a trial with DHE. The average length of follow-up was 24.7 months (standard deviation 14.5), with a minimum of 4 and a maximum of 59 months.

While in hospital, the DHE treatment provided complete relief of headache for $62(47 \%)$ of patients, more than $90 \%$ relief for $8(6 \%)$ patients, 50 to $90 \%$ relief for $11(8 \%)$ patients, 10 to $50 \%$ relief for $9(7 \%)$ patients, and no relief or worsening of headache for $40(30 \%)$ patients. 46 of the 132 patients experienced a headache free period of one month or more, with an average of 6.1 months. The average for all 132 patients having treatment was 2.1 months.

$50(40 \%)$ patients had no side effects from treatment. 15 (11\%) complained of nausea, $3(2 \%)$ had chest pain, $1(0.8 \%)$ had leg pain, $10(8 \%)$ had diarrhea, and $14(11 \%)$ had other reactions. $16(12 \%)$ had multiple side effects from treatment, and $14(11 \%)$ had the treatment terminated early because of side effects.

Figure 1 illustrates the results for headache frequency prior to DHE treatment, three months after DHE treatment, and at the time of interview.

In the three months after DHE treatment, $42(32 \%)$ patients said their headache frequency had decreased $90 \%, 19$ (14\%) said they had decreased $50 \%, 7(5 \%)$ said they decreased $25 \%$, and $63(48 \%)$ said they remained the same.

Figure 2 illustrates the results for headache intensity prior to DHE treatment, 3 months after DHE treatment, and at the time of interview.

In the three months after DHE treatment, 20 (15\%) patients said their headache intensity had decreased $90 \%, 33(25 \%)$ said they decreased $50 \%, 13(10 \%)$ had a $25 \%$ decrease in intensity, $61(46 \%)$ said their headaches remained the same in intensity, and $4(3 \%)$ said their headaches increased in severity.

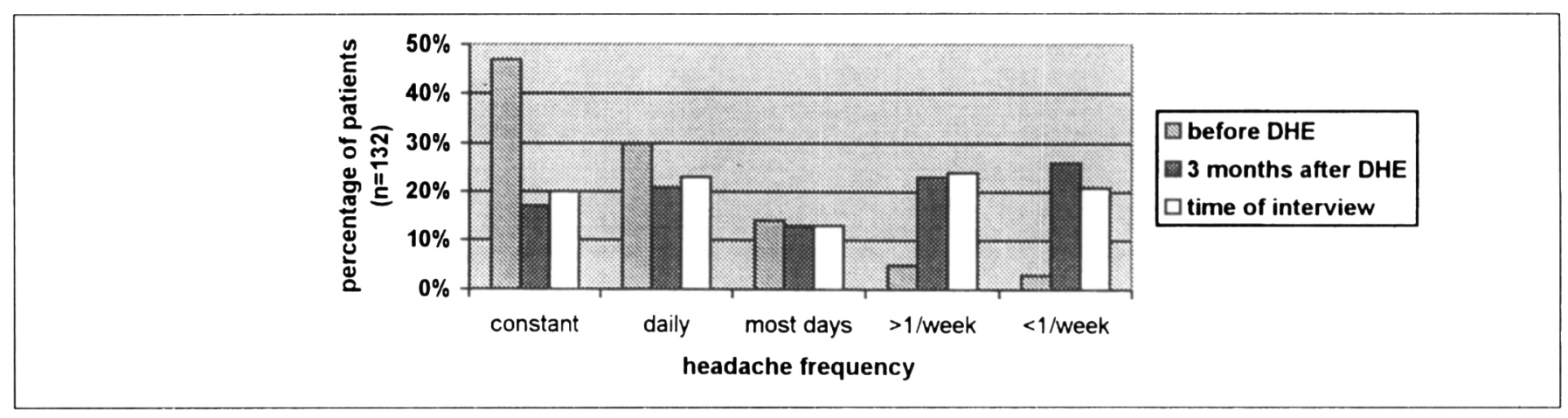

Figure 1: Headache Frequency 


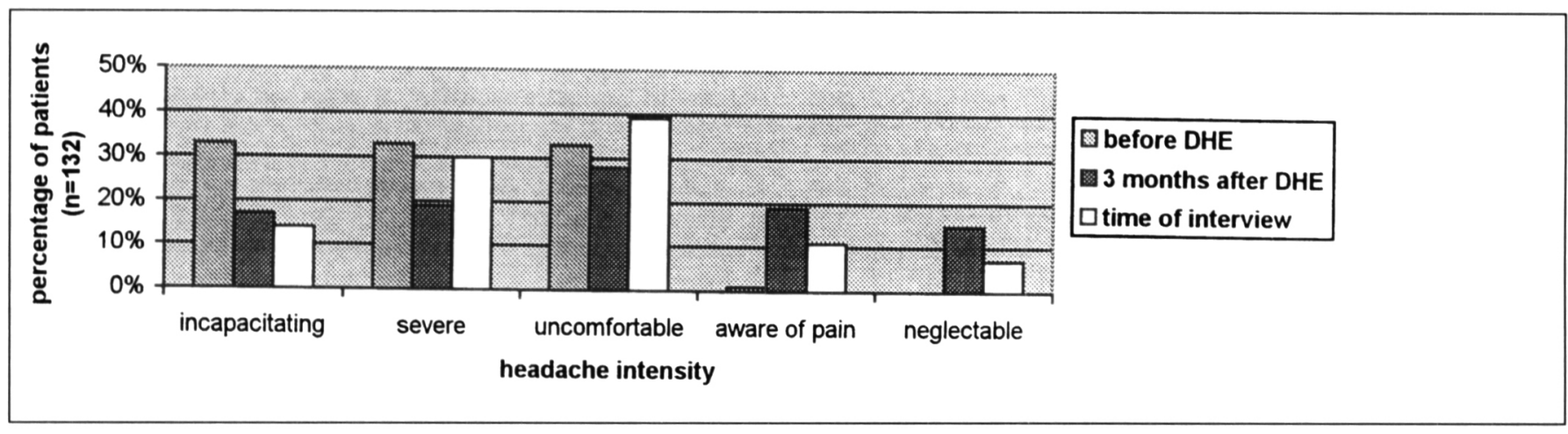

Figure 2: Headache Intensity

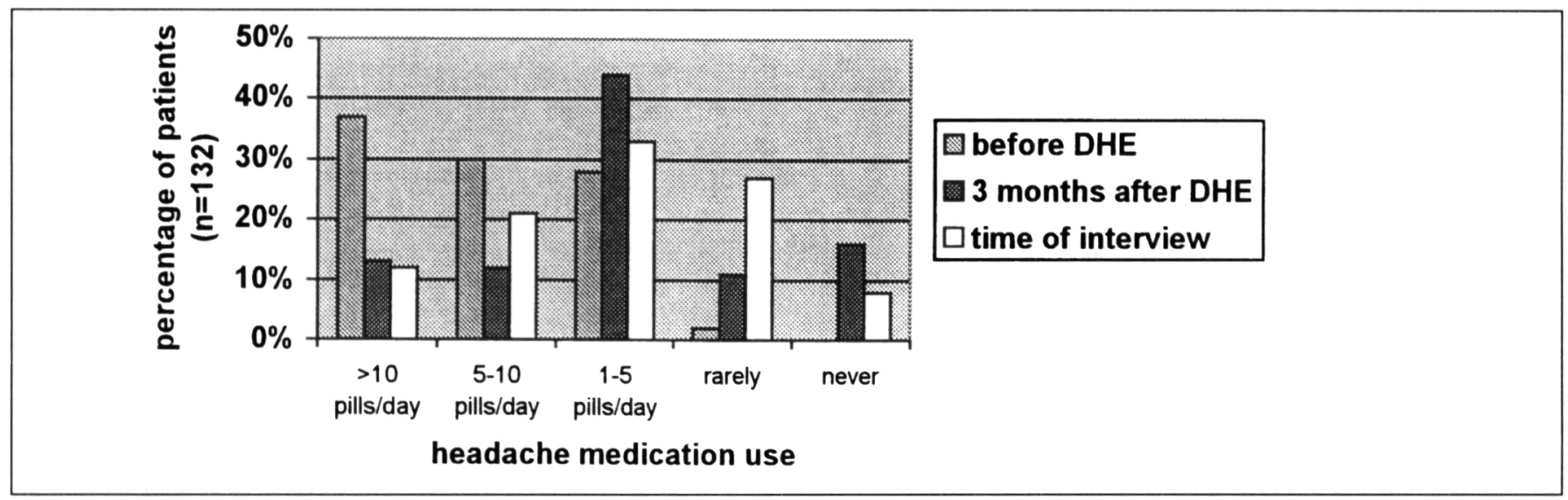

Figure 3: Headache Medication Use

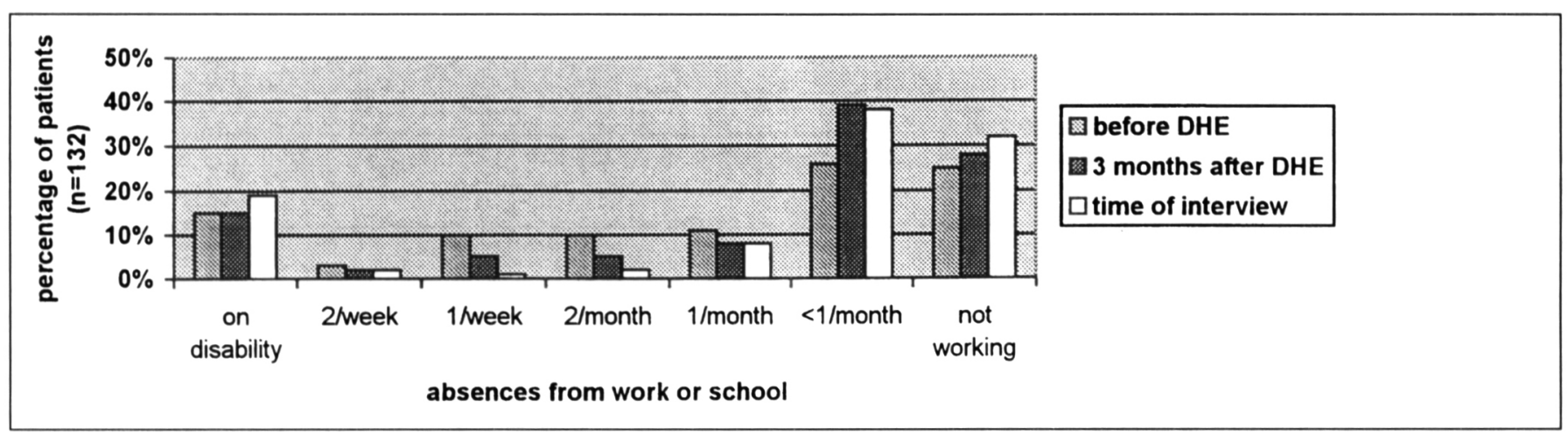

Figure 4: Absences from Work or School

Figure 3 illustrates how often patients used medications to treat their headaches prior to DHE treatment, 3 months after DHE treatment, and at the time of interview.

Since the DHE protocol, 47 (36\%) patients report that they are spending less money on headache medications, $29(22 \%)$ report that they are spending the same amount of money, $2(2 \%)$ report they are spending more money, and 54 (41\%) don't know whether they are spending more or less since the DHE protocol.

Figure 4 illustrates how often patients were absent from work or school due to a headache prior to DHE treatment, 3 months after DHE treatment, and at the time of interview.
In the three months post DHE treatment, $26(20 \%)$ patients had not been absent from work because of headaches, 20 (15\%) said their number of absences was decreased, $27(21 \%)$ said their number of absences remained the same, and $1(1 \%)$ said her number of absences increased after treatment. 37 (28\%) patients were not working outside the home, and 20 (15\%) were on disability.

We attempted to assess the impact of treatment on the patient's social and occupational functioning. In the three months post-DHE treatment, $17(13 \%)$ patients said their ability to function at work (in the home or at their work place) was 
significantly improved, $50(38 \%)$ said it was improved, and 64 (49\%) said it remained the same. In the three months after DHE treatment, $20(15 \%)$ patients said their ability to participate in recreational, social and family activities was significantly increased, 37 (28\%) patients said it was increased, $73(55 \%)$ said it remained the same, and $1(1 \%)$ said it was decreased.

When asked whether they felt the DHE protocol had made an impact on their headache profile and was worthwhile having, 59 (45\%) said yes, and $73(55 \%)$ said no. $106(80 \%)$ said they had gone through periods of depression when their headaches were severe. $90(68 \%)$ had gone to the emergency room because of their headaches.

\section{Discussion}

This study, using a retrospective phone survey, has some inherent limitations. There is the problem of recall, in that some patients were interviewed more than three years since they had the treatment, and were unlikely to remember small changes in their headache profile. The natural history of chronic daily headache also needs to be considered, although this is undocumented to our knowledge. The long-term outcome data may reflect the effect of time on the headache profile, rather than an actual treatment effect. The patients had no standardized entry criteria, and our protocol differed from that used by others particularly in that the duration of DHE treatment was only 48 hours (a decision influenced by hospital admission restrictions). Nonetheless, this study is a comprehensive survey of a group of patients with chronic daily headache, with both the longest follow-up time and largest number of patients. Our centre is a regional referral centre with no competing private neurologists, giving us accurate representation of patients with chronic daily headache in the population.

When assessing the data concerning the period prior to DHE treatment, $77 \%$ of patients had a daily or constant headache, which is expected from the criteria for treatment. These headaches were uniformly distributed between "incapacitating", "severe" and "uncomfortable", with almost no patients in the less intense categories. There was significant medication overuse, with almost $40 \%$ of patients using more than 10 pills per day to treat their headache pain. $34 \%$ of patients were forced to miss work once a month or more because of their headaches, with an additional $15 \%$ on disability because of headaches.

In the hospital, $70 \%$ of patients were able to get some relief of their headache within 48 hours. Interestingly, the rates of headache relief were higher by chart review, reflecting perhaps a communication problem between patients and health care professionals.

Nausea and diarrhea were the most common reactions to treatment, and $11 \%$ of patients did not receive full treatment due to side effects. These patients were still included in the evaluation, which may have had a negative impact on the outcome data.

By three months, more than $50 \%$ of patients maintained a reduction in their headache frequency. Whereas prior to treatment patients most commonly had a headache which was constant or daily, after treatment the headache profile evolved into a weekly pattern, with the majority of patients having headaches less than once a week, or at least once a week. This shows the success of the DHE protocol in breaking the cycle of continuous headache in some patients and returning them to an episodic migraine profile. $50 \%$ of patients experienced a decrease in the intensity of their headaches as well. A bell-shaped distribution in headache intensity is now seen. Whereas prior to treatment headaches were evenly distributed among "incapacitating", "severe" and "uncomfortable but able to carry on", there are now equal number of patients in the less intense categories of "aware of pain but not too uncomfortable" and "pain present but neglectable". There is a dramatic shift in the amount of medication used by patients at three month follow-up. Prior to treatment the majority of patients took more than ten pills per day, now, more than $40 \%$ of patients limit their medication use to 1 to 5 pills per day, with an additional $25 \%$ only rarely or never taking medication. This is in compliance with treatment recommendations to reduce medication use, and reflects the decreased need for medication because headaches are less frequent and less intense. $18 \%$ of patients continued to miss work once a month or more, compared to $34 \%$ before treatment. $50 \%$ of patients saw an improvement in their ability to function at work (in the home or at their work place), and $40 \%$ found an increase in their ability to participate in recreational, social and family activities.

The effect of treatment on the frequency of headaches appears sustained in the long-term. There is a more even distribution in headache frequency in comparison to before treatment. The effect on headache intensity is similar in the long-term, with a more bell-shaped distribution in headache intensity than prior to treatment. The decrease in analgesic use is sustained, with the majority of patients taking 1 to 5 pills per day or less to treat their headaches. The distribution of patients missing work is similar to three months after treatment, although a few more patients shifted into the disability category and a few patients retired.

The data from this study show more modest outcomes than those reported by Raskin ${ }^{5}$ and Silberstein. ${ }^{8}$ While our study found that $53 \%$ of patients had full to $90 \%$ relief of their headaches at the time of discharge, Raskin reports that $89 \%$ of his patients were discharged headache free, and Silberstein had $91 \%$ of his patients discharged headache free. There is a notable difference in their methods in comparison to ours however. The average duration of hospital stay was considerably longer, 3.8 days for Raskin, and 7.4 days for Silberstein. The hospital stay not only included the drug therapy, patients were also given classes on stress management, mechanisms of pain, family communication, medication and relaxation, as well as receiving a physical therapy evaluation and treatment program. This multidisciplinary approach to chronic daily headache management may account for the greater success of these researchers in treating patients.

No direct comparison can be made with Silberstein's longterm follow-up study of fifty patients who received DHE therapy," as he only reported on patients who initially responded to therapy, and has a much different grading scale to assess outcome.

In conclusion, nearly $50 \%$ of patients with chronic daily headache refractory to outpatient management received longterm benefit from in-patient treatment with DHE, although it is clear that it is not useful for all patients. A prospective study is needed to clarify the clinical features of responders and non responders, and to identify features that will enhance the effectiveness of the DHE protocol in this difficult group of patients.

\section{ACKNOWLEDGEMENTS}

The authors would like to acknowledge RJ Riopelle, DG Brunet, HB Dinsdale, A Jackson, and DF Weaver for their assistance in this project. and making their patients available for review, and Trish Forrest for helping with the statistics. 


\section{REFERENCES}

1. Newman LC, Lipton RB, Soloman S, Stewart WF. Daily headaches in a population sample: results from the American Migraine Study. Headache 1994; 34: 295.

2. Mathew NT. Chronic refractory headache. Neurology 1993; 43 (Suppl 3): S26-S33.

3. Silberstein SD, Lipton RB, Sliwinski M. Classification of daily and near daily headaches: field trial of revised HIS criteria. Neurology 1996; 47(4): 871-875.

4. Raskin NH. Acute and prophylactic treatment of migraine: practical approaches and pharmacologic rationale. Neurology 1993; 43 (Suppl 3); S39-S42.
5. Raskin NH. Repetitive intravenous dihydroergotamine as therapy for intractable migraine. Neurology 1986; 36: 995-997.

6. Silberstein SD. The pharmacology of ergotamine and dihydroergotamine. Headache 1997; 37 (Suppl 1): S15-S25.

7. Goadsby PJ, Edvinsson L. The trigeminovascular system and migraine: studies characterizing cerebrovascular and neuropeptide changes seen in humans and cats. Ann Neurol 1993; 33: 4856.

8. Silberstein SD, Schulman EA, McFadden-Hopkins M. Repetitive intravenous DHE in the treatment of refractory headache. Headache 1990; 30: 334-339.

9. Silberstein SD, Silberstein JR. Chronic daily headache: long-term prognosis following in-patient treatment with repetitive IV DHE. Headache 1992; 32: 439-445. 